\title{
Rib 2
}

National Cancer Institute

\section{Source}

National Cancer Institute. Rib 2. NCI Thesaurus. Code C52766.

The second rib counting from the top of the rib cage down. 